\title{
Distance-controlled scattering in a plasmonic trap
}

\author{
Lina Huang and Olivier J. F. Martin ${ }^{\text {a) }}$ \\ Nanophotonics and Metrology Laboratory, Swiss Federal Institute of Technology Lausanne (EPFL), \\ Lausanne 1015, Switzerland
}

(Received 12 August 2009; accepted 20 December 2009; published online 16 February 2010)

\begin{abstract}
Light scattered by a dielectric object when it is trapped in the field of a plasmonic nanostructure is studied theoretically and experimentally using both dielectric spheres and S. cerevisiae cells. A dramatic enhancement of the scattered light is observed for short separation distances between scatterer and plasmonic trap. It is shown that this effect can serve to selectively image cells after their immobilization and distinguish them from a turbid background. The high sensitivity of the scattered light to the separation distance and lateral displacement also provides additional insights in the configuration of the cell within the trap. (c) 2010 American Institute of Physics.
\end{abstract}

[doi:10.1063/1.3291564]

Plasmonic materials have been explored in a variety of disciplines because of their exceptional optical properties. ${ }^{1}$ By artificially tailoring the geometry of plasmonic structures, it is possible to tune their response through the optical range. Those artificially designed structures now certainly become materials of choice for optical research and have been extensively employed in the field of label-free sensing ${ }^{2,3}$ and enhanced Raman scattering; ${ }^{4-6}$ emerging applications include imaging beyond the diffraction limit $^{7}$ and cloaking. ${ }^{8}$ In this letter, we study the scattering interaction between a plasmonic nanostructure and a dielectric particle. Previous studies by a few other groups pointed out that a continuous metallic film can dramatically alter the far field scattering of small particles. Jory et al. ${ }^{9}$ measured the scattering of microscopic dielectric spheres deposited on a continuous gold film and found that the scattering is remarkably enhanced compared to the situation where the particle is directly deposited on a bare glass substrate. Lévêque et al. ${ }^{10,11}$ theoretically predicted the distance-dependant plasmon resonant coupling between a gold nanoparticle and a gold film. This effect was then experimentally verified by the group of Smith: by introducing a layer-by-layer deposition of polyelectrolyte, they were able to precisely control the separation distance between nanoparticles and film from 0.64 to $22.3 \mathrm{~nm}$. In addition, they pointed out that instead of total internal reflection illumination from the glass side, dark field illumination from the air side can also produce a scattering interaction visible in the far field. ${ }^{12}$ Similar work was reported by He et al. ${ }^{13}$ in 2004.

In this letter, we consider a system consisting of a gold disk (GD) and a latex sphere (LS). The reason to use a gold disk instead of a continuous film, is that such a disk can function as plasmonic trap integrated into the experiment. This recent trapping technique allows us to conveniently place small objects at the vicinity of the plasmonic structures without resorting to a polyelectrolyte or $\mathrm{SiO}_{2}$ spacer. ${ }^{12,13}$ As a matter of fact, the strong field gradients generated by the plasmonic nanostructure create optical forces which trap the dielectric sphere. ${ }^{14-16}$ In the following, we show both in theory and experiments that the LS will remarkably scatter visible light under dark field illumination when it approaches

${ }^{a)}$ Electronic mail: olivier.martin@epfl.ch. the GD. In parallel, we propose and demonstrate how this effect can be used to selectively light up a dielectric object in a complex environment, such as a turbid medium.

In the plasmonic trapping experiment reported in Ref. 16, we observed that when a LS passes aloft a resonant GD, it becomes rapidly trapped and at the same time appears as a very bright spot. In the experiment, an infrared laser diode $(\lambda=810 \mathrm{~nm}$, Changchun New Industries Optoelectronics Technology Co., Ltd.) is used to excite the localized plasmon modes of the GD (radius $1 \mu \mathrm{m}$ and height $40 \mathrm{~nm}$ ). Plasmonic traps are thus created to capture LS or other particles. Images are recorded with a charge coupled device camera under dark field condition (Objective: Nikon $20 \times 0.40)$ using a $50 \mathrm{~W}$ halogen lamp (Olympus) for illumination. The infrared wavelength used for trapping is suppressed with a notch filter (Semrock, NF01-808U-25). In that setup, the GD appears as a bright ring because white light is scattered by its edge. Yet, this signal is dramatically enhanced when a LS approaches on the top of the GD.

This is illustrated in Fig. 1, which shows three sequential measurements of the signal scattered by a system formed by a GD and a LS, for three different positions of the LS. The GD is deposited on a glass substrate and the LS moves from right to left in a microfluidic channel. ${ }^{16}$ The relative positions along the $x$-axis of the two scatterers are explicitly observed by plotting the normalized intensity along the dashed red lines (the background intensity is suppressed in these plots). Figure 1(a) shows the moment when the LS approaches the GD from the right: the right edge of the GD and the left edge

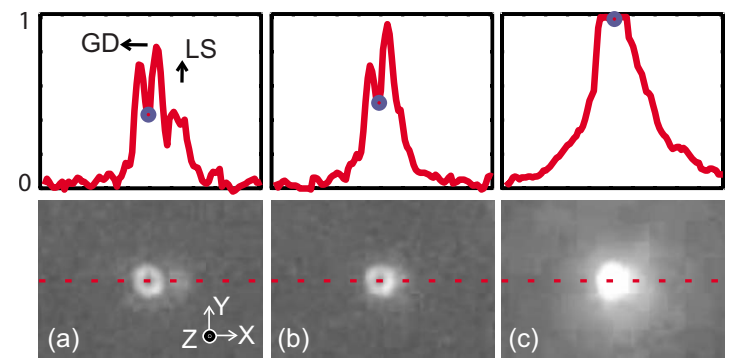

FIG. 1. (Color online) Sequential images of a LS moving along the $(-x)$-axis. (a) The LS begins to pass aloft the GD. (b) The LS almost completely overlaps the GD. (c) The plasmonic trap attracts the LS toward the GD surface and a strong scattering signal is observed. 


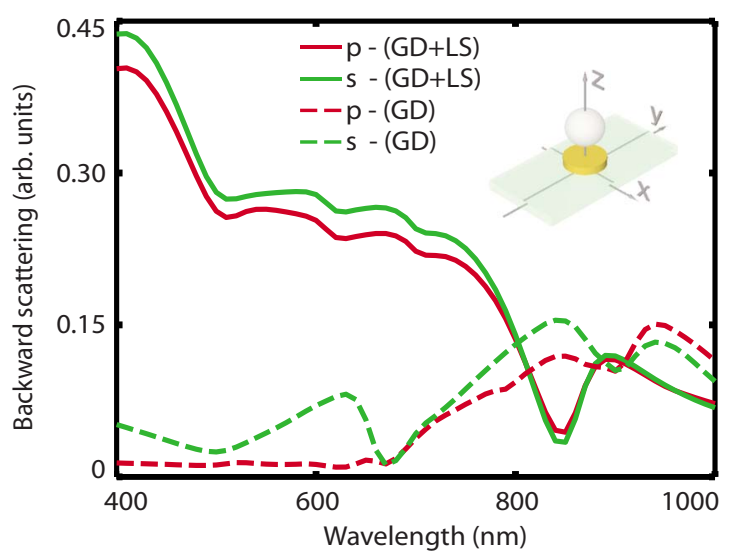

FIG. 2. (Color online) Computed backward scattered intensity spectrum for a GD+LS coupled system (solid lines) and for a single GD (dashed lines). Both illumination polarizations are considered: for p-polarized light (red lines) and s-polarized light (green lines). A remarkable enhancement of the scattered light is observed for the coupled system.

of the LS just begin to overlap. However, the LS is still far away from the GD in the $z$-direction since it is clearly out of focus in the image. Figure 1(b) shows the moment when the LS almost completely overlaps the GD. The intensity profile of the GD does not change a lot: the two peaks corresponding to the left and right edges of the GD are still clearly visible, indicating that the LS is still far away above the GD because of the profile of the laminar flow in the microfluidic channel. In this overlapping position, the plasmonic trap begins to act on the LS, which is rapidly attracted toward the surface of the GD. At this moment, the LS shines up and appears as a very bright spot, Fig. 1(c). In the following section, we investigate the distance dependence of this effect and show how to estimate the distance between the GD and the LS.

In the simulations, we first compare the light scattered by a single GD with that scattered by a coupled system consisting of a GD and a LS. All calculations include a halfspace dielectric substrate (permittivity $\epsilon=2.25$ ) covered with water, like in the experiment. The GD is deposited on the dielectric substrate; it has a $100 \mathrm{~nm}$ radius and a $40 \mathrm{~nm}$ height and its dispersive permittivity is taken from Ref. 17. The LS (permittivity 2.56), radius $100 \mathrm{~nm}$, is first located 10 $\mathrm{nm}$ above the center of the GD. In order to simulate dark field illumination as in the experiment, a plane wave illumination is used with the $k$-vector intersecting at an angle of $23.6^{\circ}$ with the $z$-axis in the second quadrant of $x z$-plane. We apply the Green's tensor technique ${ }^{18,19}$ to compute the backward scattered light along the optical axis for p-polarized and s-polarized illumination. In view of its small dimension, the LS can be discretized with a single dipole. The strong influence of the LS on the light scattered by the system is clearly visible in Fig. 2, where we compare the light scattered only by the GD (dashed line, Fig. 2) and that scattered by the system formed by the GD and the LS positioned $10 \mathrm{~nm}$ above the GD (solid line, Fig. 2) as sketched in the inset of this figure. Integrated over the visible spectrum (400-800 $\mathrm{nm})$, the coupled system scatterers 5 times more light than the GD alone. Furthermore, this effect does not depend much on the illumination polarization (red curves in Fig. 2 correspond to p-polarization, while green curves correspond to s-polarization). Hence, when a particle is close enough from the surface of a GD it will dramatically lighten up, as ob-

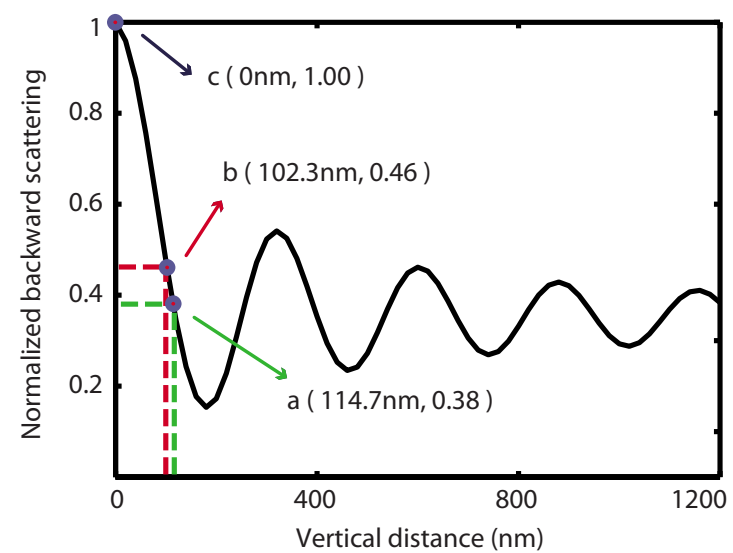

FIG. 3. (Color online) Normalized scattered intensity as a function of the vertical separation distance ( $z$-direction) for a GD+LS coupled system. The intensities measured on the three panels of Fig. 1 are shown as a, b, and c and can be used to obtain the separation distance between the GD and the LS.

served in the measurements shown in Fig. 1(c). Note however that the proximity of the particle does not modify the spectral response of the gold disk significantly.

In order to obtain a quantitative measurement of the position of the LS, we then examine the influence on the scattered intensity of the vertical separation distance $z$ between GD and LS. For simplicity we focus on s-polarization illumination, the results are similar for p-polarization. Figure 3 shows the calculation of the backward scattered light intensity for the wavelength $\lambda=600 \mathrm{~nm}$ located in the middle of the examined spectrum. When the LS moves away from the GD, this intensity oscillates periodically due to the interference between the light scattered by the GD and that scattered by the LS. A similar phenomenon was previously reported by Fischer et al. ${ }^{20}$

As we know, the scattered field $E_{\text {scat }}$ can be written as $E_{\text {scat }}=\alpha E^{\circ}$, where $\alpha$ is the system polarizability and $E^{\circ}$ the incident field. Since the polarizability is proportional to the volume $v$ of the system, the total scattered field can be scaled with that volume. From Fig. 1 we have measured the normalized scattered intensity of a single GD, and of a coupled $\mathrm{GD}+\mathrm{LS}$ system. Values are taken from the blue points as $I_{a}=0.38, I_{b}=0.46, I_{c}=1.00$. We assume that $I_{a}$ is the scattered intensity when the LS is located far away from the GD surface. From Fig. 3, this distance is estimated to be 114.7 $\mathrm{nm} . I_{b}$ is the scattered intensity measured when the LS is located on top of the GD, where the plasmonic trapping begins to act. At this moment, the separation distance is found to be $102.3 \mathrm{~nm}$ from Fig. 3. Finally, $I_{c}$ represents the total scattering of the coupled system, when the plasmonic trap brings the LS at the very close vicinity of the GD. Their separation distance is estimated to be almost zero.

Let us finally examine the influence of the lateral displacement in $x$-direction between the GD and the LS. We again focus on s-polarization, but also include now the spectral response of the system. Figure 4 shows the scattered intensity when the LS moves from $x=-300 \mathrm{~nm}$ to $300 \mathrm{~nm}$, at a constant height $z=10 \mathrm{~nm}$ above the GD surface. Unsurprisingly, we find that the scattered intensity is dramatically enhanced when the LS is located above the GD.

This strong enhancement of scattered signal in a plasmonic trap can be very useful for applications in chemistry and biology. Not only can a particle or a cell be immobilized 


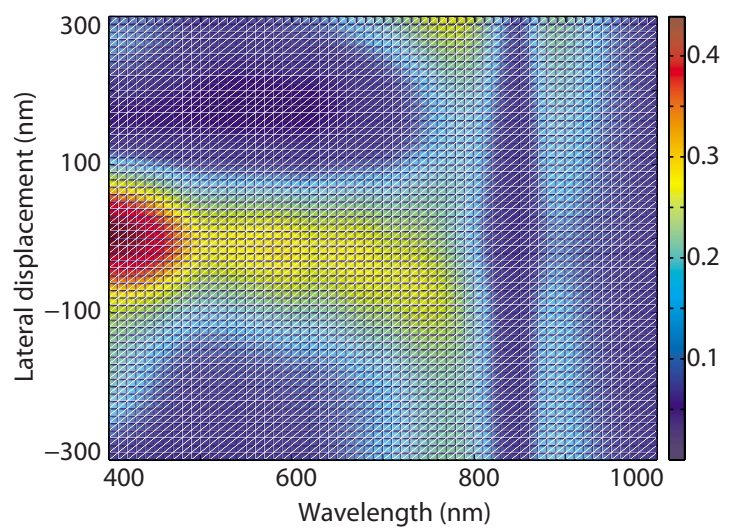

FIG. 4. (Color online) Scattered intensity spectrum as a function of the lateral separation distance ( $x$-direction) between the GD and the LS. The LS is kept at a constant height $z=10 \mathrm{~nm}$ above the GD. The scattering is strongly enhanced when the LS overlaps the GD.

with great convenience, but also this entity can be easily identified from a turbid background thanks to the strong optical signal it then generates. To demonstrate this, we choose $S$. cerevisiae as it is one of the dominant model organisms in use today. In Fig. 5, particle A and C are S. cerevisiae cells trapped on top of GDs; one single GD traps one single cell. Particle B represents a single GD without trapped cells and its signal serves as reference. By comparing the scattered intensity for the different traps, we are able to determine that

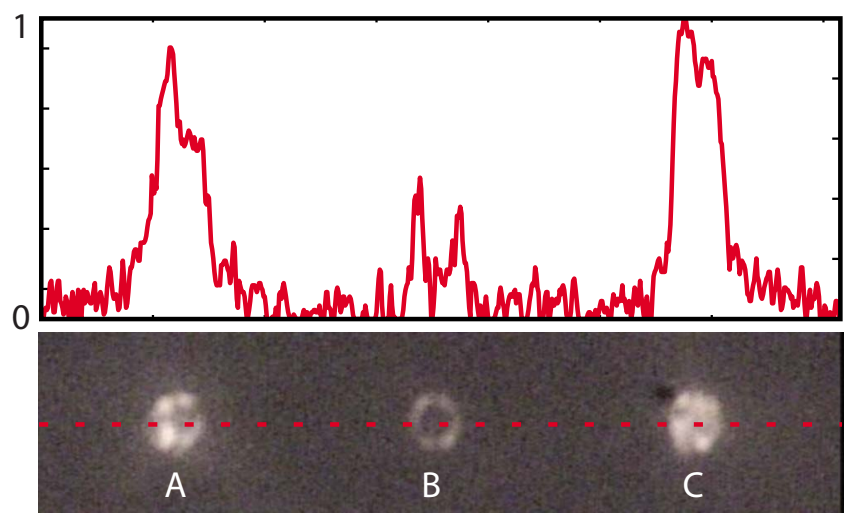

FIG. 5. (Color online) Plasmonic trap enhanced scattering. In A and $\mathrm{C}$ a $S$. cerevisiae cell is trapped on top of a GD; no cells are trapped in $\mathrm{B}$. The relative scattered intensity in the different situations gives an indication of the distance between the $S$. cerevisiae cell and the GD. cell A is further away from the GD surface than cell C; even though both of them are trapped.

As conclusion, we have numerically studied the distance-dependent enhanced scattering from a metaldielectric coupled system. Experimentally, we have used the specific trapping properties of this system to bring the dielectric object at the intimate vicinity of the metallic one; and by measuring the backward scattered intensity, we were able to estimate the separation distance between the particles. This combined plasmonic trapping and dark field scattering technique can serve as an alternative method for imaging cells after their immobilization; its high sensitivity to separation distances and lateral displacements can provide additional insights in the configuration of the cell within the trap.

We are grateful to J. Dintinger and S. J. Maerkl for experimental support, to $\mathrm{Y}$. Gu for stimulating discussions, and to the Swiss National Science Foundation for funding (Grant No. 200021-113735).

${ }^{1}$ W. L. Barnes, A. Dereux, and T. W. Ebbesen, Nature (London) 424, 824 (2003).

${ }^{2}$ J. Homola, Surface Plasmon Resonance Based Sensors (Springer, Berlin, 2006).

${ }^{3}$ A. V. Whitney, J. W. Elam, S. L. Zou, A. V. Zinovev, P. C. Stair, G. C. Schatz, and R. P. Van Duyne, J. Phys. Chem. B 109, 20522 (2005).

${ }^{4}$ S. J. Oldenburg, S. L. Westcott, R. D. Averitt, and N. J. Halas, J. Chem. Phys. 111, 4729 (1999).

${ }^{5}$ K. Kneipp, Y. Wang, H. Kneipp, L. T. Perelman, I. Itzkan, R. Dasari, and M. S. Feld, Phys. Rev. Lett. 78, 1667 (1997).

${ }^{6}$ A. Rasmussen and V. Deckert, J. Raman Spectrosc. 37, 311 (2006).

${ }^{7}$ N. Fang, H. Lee, C. Sun, and X. Zhang, Science 308, 534 (2005).

${ }^{8}$ W. S. Cai, U. K. Chettiar, A. V. Kildishev, and V. M. Shalaev, Nat. Photonics 1, 224 (2007).

${ }^{9}$ M. J. Jory, P. S. Cann, J. R. Sambles, and E. A. Perkins, Appl. Phys. Lett. 83, 3006 (2003).

${ }^{10}$ G. Lévêque and O. J. F. Martin, Opt. Express 14, 9971 (2006).

${ }^{11}$ G. Lévêque and O. J. F. Martin, Opt. Lett. 31, 2750 (2006).

${ }^{12}$ J. J. Mock, R. T. Hill, A. Degiron, S. Zauscher, A. Chilkoti, and D. R. Smith, Nano Lett. 8, 2245 (2008).

${ }^{13}$ L. He, E. A. Smith, M. J. Natan, and C. D. Keating, J. Phys. Chem. B 108, 10973 (2004).

${ }^{14}$ M. Righini, A. S. Zelenina, C. Girard, and R. Quidant, Nat. Phys. 3, 477 (2007).

${ }^{15}$ X. Y. Miao and L. Y. Lin, IEEE J. Sel. Top. Quantum Electron. 13, 1655 (2007).

${ }^{16}$ L. Huang, S. J. Maerkl, and O. J. F. Martin, Opt. Express 17, 6018 (2009).

${ }^{17}$ P. B. Johnson and R. W. Christy, Phys. Rev. B 6, 4370 (1972).

${ }^{18}$ O. J. F. Martin and N. B. Piller, Phys. Rev. E 58, 3909 (1998).

${ }^{19}$ M. Paulus and O. J. F. Martin, J. Opt. Soc. Am. A Opt. Image Sci. Vis. 18, 854 (2001).

${ }^{20}$ H. Fischer and O. J. E. Martin, Opt. Lett. 34, 368 (2009). 